\title{
Antioxidant Treatment Induces Hyperactivation of the HPA Axis by Upregulating ACTH Receptor in the Adrenal and Downregulating Glucocorticoid Receptors in the Pituitary
}

\author{
Jessika P. Prevatto, ${ }^{1}$ Rafael C. Torres, ${ }^{2}$ Bruno L. Diaz, ${ }^{2}$ Patrícia M. R. e Silva, ${ }^{1}$ \\ Marco A. Martins, ${ }^{1}$ and Vinicius F. Carvalho ${ }^{1,3}$ \\ ${ }^{1}$ Laboratório de Inflamação, Instituto Oswaldo Cruz, Fundação Oswaldo Cruz, Av. Brasil, No. 4365, Manguinho, $21045-900$ Rio de \\ Janeiro, RJ, Brazil \\ ${ }^{2}$ Laboratório de Inflamação, Instituto de Biofísica Carlos Chagas Filho, Universidade Federal do Rio de Janeiro, Av. Carlos Chagas \\ Filho, No. 373, Cidade Universitária-Ilha do Fundão, 21941-902 Rio de Janeiro, RJ, Brazil \\ ${ }^{3}$ National Institute of Science and Technology on Neuroimmunomodulation (INCT-NIM), Rio de Janeiro, RJ, Brazil
}

Correspondence should be addressed to Vinicius F. Carvalho; viniciusfrias@hotmail.com

Received 10 January 2017; Revised 10 March 2017; Accepted 20 March 2017; Published 21 May 2017

Academic Editor: Ayman M. Mahmoud

Copyright (c) 2017 Jessika P. Prevatto et al. This is an open access article distributed under the Creative Commons Attribution License, which permits unrestricted use, distribution, and reproduction in any medium, provided the original work is properly cited.

Glucocorticoid (GC) production is physiologically regulated through a negative feedback loop mediated by the GC, which appears disrupted in several pathological conditions. The inability to perform negative feedback of the hypothalamus-pituitary-adrenal (HPA) axis in several diseases is associated with an overproduction of reactive oxygen species (ROS); however, nothing is known about the effects of ROS on the functionality of the HPA axis during homeostasis. This study analyzed the putative impact of antioxidants on the HPA axis activity and GC-mediated negative feedback upon the HPA cascade. Male Wistar rats were orally treated with $\mathrm{N}$-acetylcysteine (NAC) or vitamin $\mathrm{E}$ for 18 consecutive days. NAC-treated rats were then subjected to a daily treatment with dexamethasone, which covered the last 5 days of the antioxidant therapy. We found that NAC and vitamin $\mathrm{E}$ induced an increase in plasma corticosterone levels. NAC intensified MC2R and StAR expressions in the adrenal and reduced GR and MR expressions in the pituitary. NAC also prevented the dexamethasone-induced reduction in plasma corticosterone levels. Furthermore, NAC decreased HO-1 and Nrf2 expression in the pituitary. These findings show that antioxidants induce hyperactivity of the HPA axis via upregulation of MC2R expression in the adrenal and downregulation of GR and MR in the pituitary.

\section{Introduction}

Reactive oxygen species (ROS) are ions or small molecules containing oxygen and an unpaired electron, and this free electron confers high reactivity to oxygen. ROS production in mammals is due to the activity of endogenous pro-oxidant enzymes NADPH oxidase, xanthine oxidase, peroxisomes, and cytochrome P-450. Their product is counterbalanced by endogenous antioxidant enzymes including superoxide dismutase, catalase, glutathione peroxidase, reduced glutathione, and heme oxygenase- (HO-) 1 [1]. These antioxidant defense systems are directly regulated by nuclear factor erythroid 2related factor 2 (Nrf2). Besides inducing the transcription of endogenous antioxidant enzymes, Nrf2 affects the homeostasis of ROS and reactive nitrogen species (RNS) through regeneration of oxidized cofactors and proteins; synthesis of reducing factors, as GSH and NADPH; and increasing redox transport, including cysteine/glutamate transport by $\mathrm{xCT}$ [2]. Redox imbalance is induced by disequilibrium between the production and suppression of ROS. Furthermore, excess of oxidative damage can be controlled by exogenous antioxidants such as vitamins $\mathrm{C}$ and $\mathrm{E}$, polyphenols, carotenes, flavonoids, omega-3, and N-acetylcysteine (NAC) [3-5]. These exogenous antioxidants decrease the oxidative damage through distinct mechanisms of action. For instance, vitamin $\mathrm{E}$, a nonenzymatic antioxidant, promotes a lipid 
peroxyl radical scavenger and maintains the integrity of long-chain polyunsaturated fatty acids in the membrane of cells $[6,7]$, while NAC, which is an acetylated cysteine residue, stimulates glutathione synthesis and performs as a scavenger of oxygen free radicals [8].

The HPA axis is a neuroendocrine system regulated by the circadian cycle $[9,10]$ and stress [11]. After being stimulated, neurons of the paraventricular nucleus of the hypothalamus release corticotropin-releasing hormone $(\mathrm{CRH})$, which will be transported by hypothalamic-pituitary portal circulation and stimulate pituitary corticotroph cells to cleave proopiomelanocortin (POMC) in adrenocorticotrophic hormone (ACTH) [12]. ACTH is released into the bloodstream and acts on melanocortin receptor type 2 (MC2R), situated in the zona fasciculata of adrenals, inducing an increase in expression and/or activity of steroidogenic enzymes, including $11 \beta$ hydroxysteroid dehydrogenase-type 1 (11 $\beta$-HSD1) and steroidogenic acute regulatory protein (StAR), and releasing glucocorticoids to the bloodstream [13]. The basal activity of the HPA axis is regulated by a negative feedback which is mediated through activation of glucocorticoid receptor (GR) and mineralocorticoid receptor (MR), located in the pituitary and hypothalamus, by glucocorticoids $[14,15]$.

The HPA axis is the main neuroendocrine system that regulates responses to stress. The production of high levels of ROS into the glands that comprise the HPA axis is associated with the activation of a stress-response system in several models of stress, including social isolation [16] and inflammatory and infectious diseases [17]. HPA axis hyperactivity induced by redox imbalance may occur by a reduction in negative feedback through a decrease in GR translocation to the cellular nucleus in corticotroph cells of the pituitary [17].

Although the free radical theory and the oxidative damage theory describe that accumulation of oxidative damage in cellular macromolecules is immensely toxic, ROS products by normal cell metabolism are vital to cell homeostasis maintenance, especially for its roles in immunocompetence and activation of several signal transduction pathways $[18,19]$. Indeed, the antioxidant therapy presents several side effects, which is opposed to the anticipated properties of these substances [3]. Our hypothesis is that any imbalance in the redox system alters the homeostasis of the HPA axis culminating in its hyperactivation, and not just an increase in ROS production as shown in several diseases. Here, we undertook this study to evaluate the putative mechanism underlying the antioxidant-induced hyperactivation of the HPA axis in Wistar rats.

\section{Materials and Methods}

2.1. Animals and Treatments. Male Wistar rats (250-300 g) were obtained from the Oswaldo Cruz Foundation breeding colony and used in accordance with the guidelines of the Committee on Use of Laboratory Animals of the Oswaldo Cruz Foundation (CEUA-FIOCRUZ, license LW-23/11). Rats were housed in groups of three in a temperature-, humidity-, and light-controlled ( $12 \mathrm{~h}$ light: $12 \mathrm{~h}$ darkness cycle) colony room. Rats were given ad libitum access to food and water.
Twelve male rats were randomly assigned into 2 groups as follows: control $(n=6)$ and treated with the antioxidant $\mathrm{N}$ acetyl L-cysteine (NAC) $(n=6)$. In another set of experiments, twelve male rats were randomly assigned into 2 groups as follows: control $(n=6)$ and treated with the antioxidant vitamin $\mathrm{E}(n=6)$. In a third set of experiments, twenty male rats were randomly divided into 4 experimental groups: control $(n=5)$, treated with NAC $(n=5)$, treated with the exogenous glucocorticoid dexamethasone $(n=5)$, and treated with NAC $(n=5)$ plus dexamethasone. The rats were treated with NAC (150 mg/kg body weight) [20] or vitamin E ( $\alpha$ tocopherol, $40 \mathrm{mg} / \mathrm{kg}$ body weight) [21] by gavage once a day, during 18 consecutive days. Control rats received an equal volume of vehicle (sterile saline $0.9 \%$ and DMSO $0.1 \%$, resp.). To analyze corticoid-induced negative feedback sensitivity, a group of animals received dexamethasone $(0.02 \mathrm{mg} / \mathrm{kg}$ body weight, s.c.) daily, for 5 consecutive days [22], beginning 13 days after the starting of antioxidant treatment.

2.2. Corticosterone Quantification. Animals were euthanized in a $\mathrm{CO}_{2}$ chamber, during the nadir $(08: 00 \mathrm{~h})$ of the circadian rhythm as described previously [23], and the blood was immediately collected from the abdominal aorta with heparinized $(400 \mathrm{U} / \mathrm{ml})$ saline. Plasma was obtained after sample centrifugation for $10 \mathrm{~min}$ at $1000 \times \mathrm{g}$ and stored at $-20^{\circ} \mathrm{C}$ until use. Plasma corticosterone levels were detected by radioimmunoassay (RIA) following manufacturer's guidelines (MP Biomedicals, Solon, OH, USA). Briefly, this is a competitive assay between the hormone presented in the sample and the hormone labelled with radioisotope $\left(\mathrm{I}^{125}\right)$ to bind to a specific antibody. Thereby, an increase in amount of the hormone in the sample leads to a corresponding decrease in the fraction of labelled hormone bound to the antibody. Radioactivity quantification was carried out using a gamma counter (ICN Isomedic 4/600 HE; ICN Biomedicals Inc., Costa Mesa, CA, USA), and the amount of corticosterone in samples was calculated by interpolation to a standard curve performed in parallel.

2.3. Immunohistochemistry Staining. The adrenals and pituitary glands were immediately dissected after perfusion of rats with $0.9 \%$ sterile saline. Adrenals were quickly removed from the rats and cleaned of surrounding fat, while the pituitary glands were gently collected after the decapitation of rats and removal of the brain $[24,25]$. Instantly after dissection, the glands were fixed in Milloning and embedded in paraffin. Paraffin-embedded sections of $3 \mu \mathrm{m}$ of rat pituitary and adrenals were deparaffinized with xylene, rehydrated by a graded series of ethanol washes, and boiled in sodium citrate buffer ( $10 \mathrm{mM}, \mathrm{pH} 6.0$ ) at the temperature of $95^{\circ} \mathrm{C}$ for $15 \mathrm{~min}$ to enhance antigen retrieval. Tissue sections were incubated with $3 \% \mathrm{H}_{2} \mathrm{O}_{2}$ in methanol for 20 min to block endogenous peroxidases. To prevent nonspecific binding, sections were then incubated for $3 \mathrm{~h}$ with a solution containing $2.5 \%$ bovine serum albumin (BSA), $8 \%$ fetal bovine serum (FBS), and $1 \%$ of nonfat milk dissolved in Tris-buffered saline enriched with $0.1 \%$ Tween 20 (TBST). After blocking, sections were incubated with primary specific antibody (polyclonal rabbit 


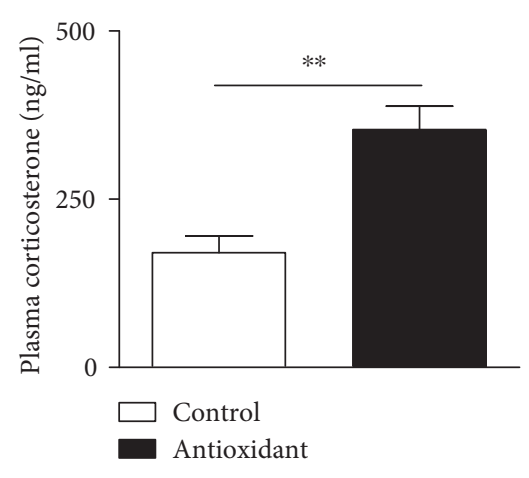

(a)

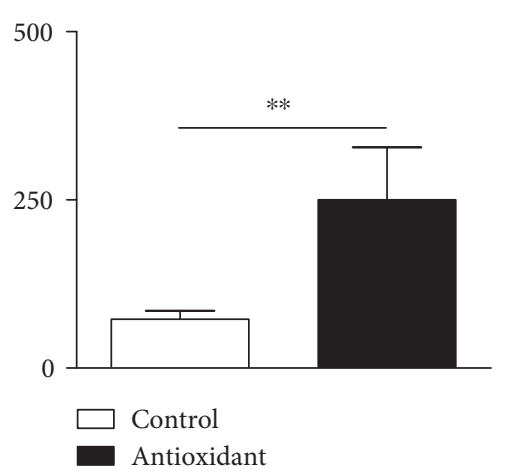

(b)

FIgURe 1: Antioxidant treatment increases circulating levels of plasma corticosterone in Wistar rats. (a) NAC (150 mg/kg, oral route) and (b) vitamin E ( $40 \mathrm{mg} / \mathrm{kg}$, oral route) were given daily for 18 consecutive days. Untreated animals received an equal amount of vehicle (saline $0.9 \%$ or DMSO 0.1\%). Data are expressed as the mean \pm SEM of 6 animals. This result is a representative of two independent assays. ${ }^{* *} p<0.01$.

anti-rat StAR $(1: 50)$, GR $(1: 250)$, or MC2R $(1: 250)$ and polyclonal goat anti-rat MR $(1: 50), \mathrm{HO}-1(1: 100)$, or Nrf2 $(1: 100)$ from Santa Cruz Biotechnology, Santa Cruz, CA, USA) diluted in TBST with $1 \%$ BSA overnight at $4^{\circ} \mathrm{C}$.

Then, primary antibody binding was detected after incubating sections with a horseradish peroxidase conjugatedsecondary antibody (polyclonal anti-goat or anti-rabbit IgG HRP, R\&D System, Minneapolis, MN, USA) for $2.5 \mathrm{~h}$, followed by a $20 \mathrm{~min}$ exposure to the HRP substrate 3amino-9-ethylcarbazole (AEC). Sections were washed with TBST between all steps and weakly counterstained with hematoxylin for the easy identification of tissue structures. Finally, tissue sections were mounted in aqueous medium and images digitized via scanner microscope (Pannoramic SCAN150, 3D Histech, Budapest, Hungary) using a 20x objective lens. Images obtained from the anterior pituitary or zona fasciculata of the adrenal cortex were analyzed with Image Pro Plus 6.2 software (Media Cybernetics). Briefly, red to brown colored pixels associated with a positive immunohistochemistry stain were selected in a model image and applied to the remaining fields. The number of positive pixels was divided by the field area and expressed as pixels $/ \mu \mathrm{m}^{2}$.

2.4. Chemicals. Sodium citrate, AEC, NAC, vitamin E, dexamethasone, and hydrogen peroxide were purchased from Sigma Chemical Co. (Saint Louis, MO, USA); ethanol, methanol, and xylene from Merck (Rio de Janeiro, RJ, Brazil); and sodium heparin from Roche (São Paulo, SP, Brazil). All solutions were freshly prepared immediately before use.

2.5. Statistical Analysis. The data are reported as the mean \pm standard error of the mean (SEM). All data were evaluated to ensure normal distribution. The assay of corticoidinduced negative feedback sensitivity was analyzed by oneway ANOVA followed by a Student-Newman-Keuls post hoc test, while all the other results were statistically analyzed by unpaired $t$-test, with Graphpad Prism 5.0. Probability values $(p)$ of 0.05 or less were considered significant.

\section{Results}

3.1. Antioxidant Therapy Increases Plasma Corticosterone Levels in Wistar Rats. Initially, we investigated the impact of antioxidant therapy on circulating corticosterone levels. We observed that rats treated with either NAC (Figure 1(a)) or vitamin E (Figure 1(b)), for 18 consecutive days, presented a significant increase in plasma corticosterone levels compared to controls (mean \pm SEM, $n=6$; $p<0.01$; two-tailed $t$-test).

3.2. NAC Induces Adrenal Hypertrophy and Upregulation of ACTH Receptor and StAR in the Adrenal Cortex of Wistar Rats. We hypothesized that the high corticosterone levels were due to increased stimulation of the adrenal cortex. We noted that NAC induced adrenal hypertrophy, as evidenced by the ratio between adrenal weight $(\mathrm{mg})$ and body weight (g). The values of adrenal/body weight ratio increased from $0.066 \pm 0.003$ in control rats to $0.095 \pm 0.008$ (mean \pm SEM, $n=6 ; p<0.01$; two-tailed $t$-test) in NAC-treated rats. The absolute adrenal weights were $24 \pm 1.8 \mathrm{mg}$ and $30 \pm 1.5 \mathrm{mg}$ (mean \pm SEM, $n=6 ; p<0.05$; two-tailed $t$-test) to control and NAC-treated rats, respectively. In parallel, we showed that treatment with NAC increased the expression of ACTH receptor (MC2R) (Figures 2(b) and 2(e)) and steroidogenic enzyme StAR (Figures 2(d) and 2(f)) in the zona fasciculata of the adrenal cortex compared to that of control rats (Figures 2(a) and 2(c), resp.) (mean \pm SEM, $n=6 ; p<0.05$ and $p<0.01$, resp.; two-tailed $t$-test). The expression of MC2R is located in the membrane and cytoplasm of cells (Figures 2(a) and 2(b)), while StAR is expressed only in the cytoplasm of the cells (Figures 2(c) and 2(d)).

3.3. NAC Decreases Nrf2 and HO-1 Expression in the Anterior Pituitary of Wistar Rats. Our next approach was to determine if the treatment with NAC could interfere with the expression of antioxidant arsenal in the anterior pituitary, an important component of the HPA axis which regulates corticosterone production by adrenals. Treatment with NAC reduces the expression of transcription factor $\mathrm{Nrf2}$ (Figures 3(b) and 3(e)) and the antioxidant enzyme HO-1 


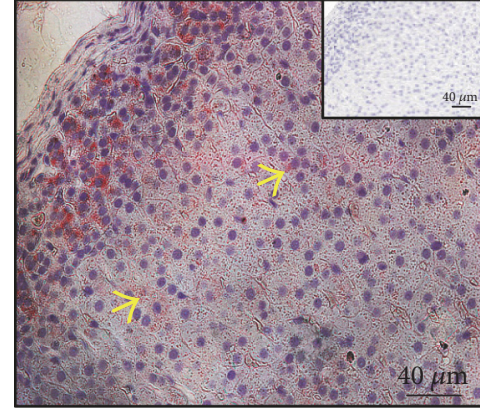

(a)

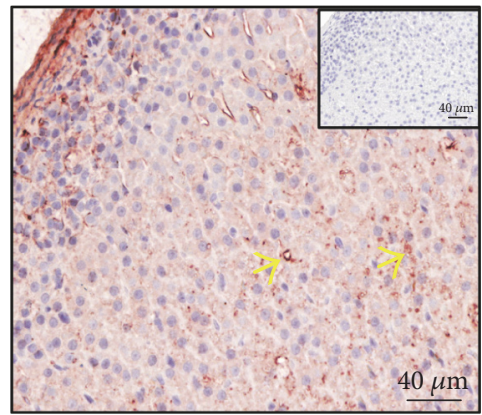

(c)

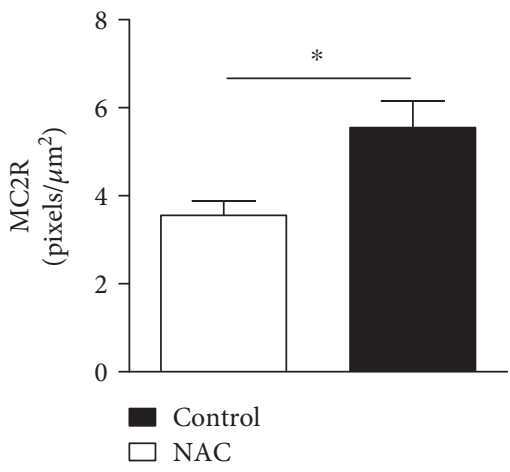

(e)

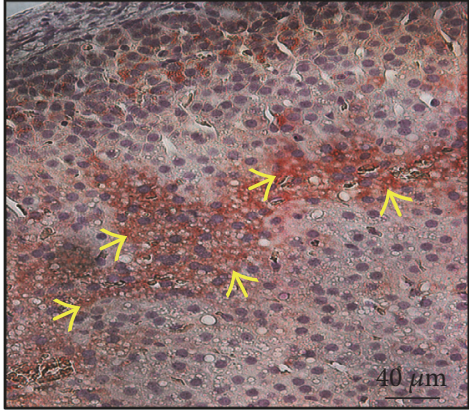

(b)

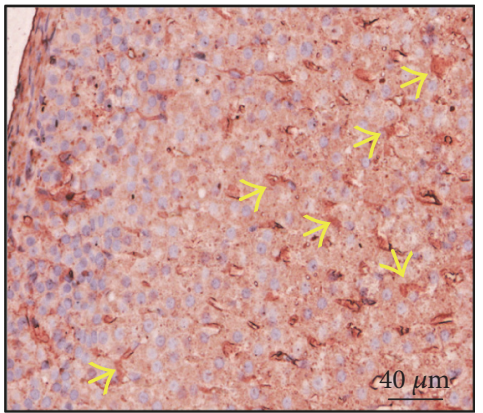

(d)

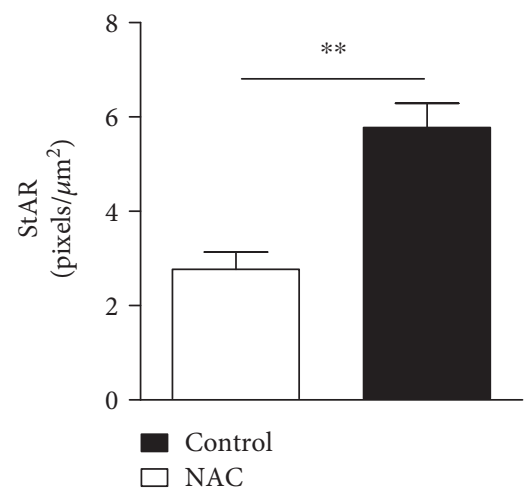

(f)

FIgURE 2: NAC induces an upregulation of MC2R and StAR expression in the zona fasciculata of the adrenal of Wistar rats. NAC (150 mg/kg, oral route) was given daily for 18 consecutive days and the analysis was made by immunohistochemistry. The panels show representative photomicrographs of adrenal expression of MC2R in the (a) control and (b) NAC-treated rats and StAR in (c) control and (d) NACtreated rats. The quantification of pixels associated with MC2R and StAR expression is shown in (e) and (f), respectively. Inserts represent negative controls. Yellow arrows indicate immunolabelling of MC2R (a, b) and StAR (c, d) in the zona fasciculata of adrenals. Data are expressed as the mean \pm SEM of 6 animals. This result is a representative of two independent assays. ${ }^{*} p<0.05$ and ${ }^{* *} p<0.01$.

(Figures 3(d) and 3(f)) in the anterior pituitary compared to that of control rats (Figures 3(a) and 3(c), resp.) (mean \pm SEM, $n=6 ; p<0.05$ and $p<0.05$, resp; two-tailed $t$-test). The expression of Nrf2 is located in the nucleus and cytoplasm of cells (Figures 3(a) and 3(b)), while HO-1 is expressed only in the cytoplasm of the cells (Figures 3(c) and 3(d)).

3.4. NAC Reduces Glucocorticoid Receptor Expression in the Anterior Pituitary and Impaired Negative Feedback of the HPA Axis in Wistar Rats. High levels of plasma corticosterone induced by antioxidants can also be associated with a failure in the negative feedback of the HPA axis. Treatment with NAC decreases the expression of both glucocorticoid receptors GR (Figures 4(b) and 4(e)) and MR (Figures 4(d) and $4(\mathrm{f}))$ in the anterior pituitary compared to that of control rats (Figures 4(a) and 4(c), resp.) (mean \pm SEM, $n=6$; $p<0.05$ and $p<0.001$, resp.; two-tailed $t$-test). The expressions of GR (Figures 4(a) and 4(b)) and MR (Figures 4(c) and $4(d))$ are located in the nucleus and cytoplasm of cells.

Thus, we treated rats with a low dose of dexamethasone $(0.02 \mathrm{mg} / \mathrm{kg}$, s.c.) and analyzed the circulating levels of corticosterone. We showed that dexamethasone induced a strong negative feedback response and reduced the plasma corticosterone levels in control rats (mean \pm SEM, $n=6 ; p<0.05$; one-way ANOVA followed by a Student-Newman-Keuls post hoc test); however, although treatment with NAC 


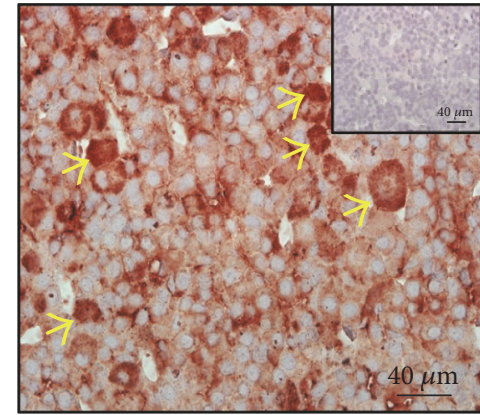

(a)

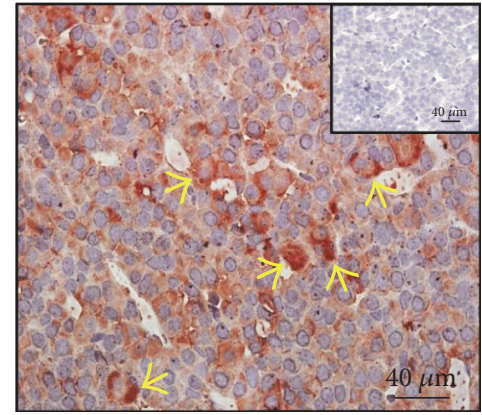

(c)

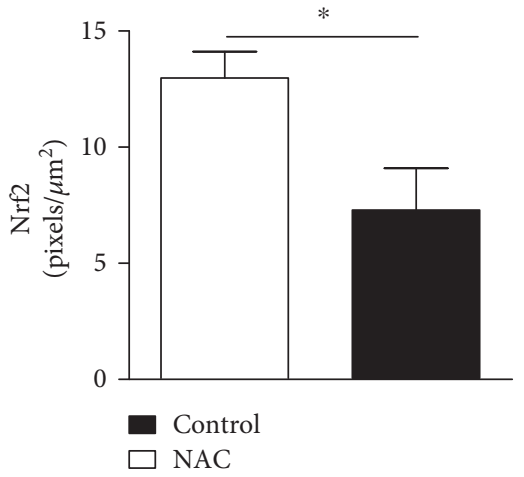

(e)

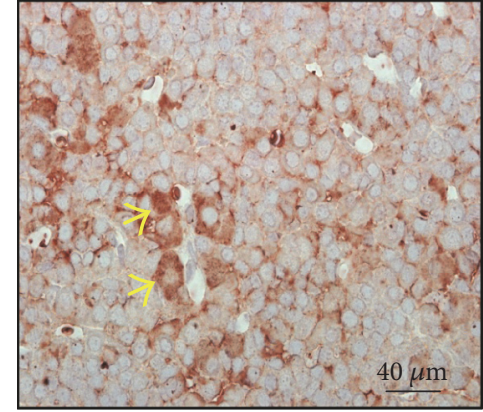

(b)

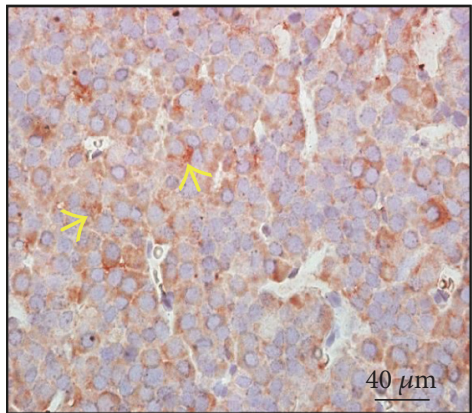

(d)

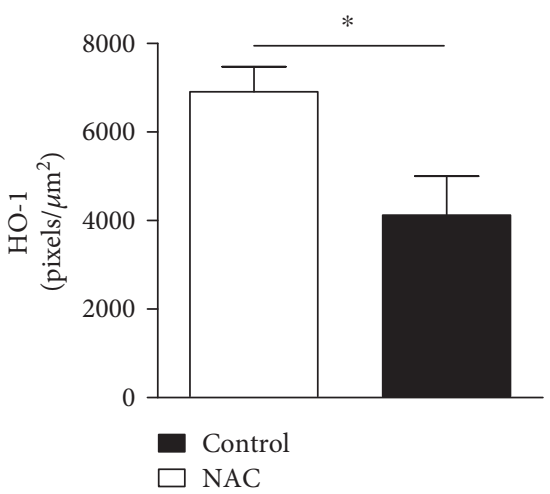

(f)

FIGURE 3: NAC reduces Nrf2 and HO-1 expression in the anterior pituitary of Wistar rats. NAC (150 mg/kg, oral route) was given daily for 18 consecutive days and analysis was made by immunohistochemistry. The panels show representative photomicrographs of pituitary expression of Nrf2 in (a) control and (b) NAC-treated rats and HO-1 in (c) control and (d) NAC-treated rats. The quantification of pixels associated with Nrf2 and HO-1 expression is shown in (e) and (f), respectively. Inserts represent negative controls. Yellow arrows indicate immunolabelling of Nrf2 (a, b) and HO-1 (c, d) in the anterior pituitary. Data are expressed as the mean \pm SEM of 6 animals. This result is a representative of two independent assays. ${ }^{*} p<0.05$.

induced an increase in plasma corticosterone levels compared to controls (mean \pm SEM, $n=6 ; p<0.01$; one-way ANOVA followed by a Student-Newman-Keuls post hoc test), dexamethasone did not alter the levels of corticosterone in NAC-treated rats (Figure 5).

\section{Discussion}

This study investigated the role of antioxidants on the modulation of endogenous glucocorticoid levels. We found that treatment with antioxidants either NAC or vitamin E increases the plasma levels of corticosterone in rats, in association with an overexpression of ACTH receptor and the steroidogenic enzyme StAR in the adrenal glands. NAC also induces a drop in HO-1 and Nrf2 expression in the pituitary and blocked the ability of dexamethasone to perform negative feedback of the HPA axis by decreasing the expression of glucocorticoid receptors in the pituitary. Our findings suggest that antioxidants cause a hyperactivation of the HPA axis with a clear dependency of upregulation of ACTH receptor in adrenals and downregulation of glucocorticoid receptors in the pituitary.

In this study, we showed that both NAC and vitamin E increase circulating levels of corticosterone in rats. NAC and vitamin $\mathrm{E}$ are antioxidants that act through distinct mechanisms of action. While NAC provides cysteine, which 


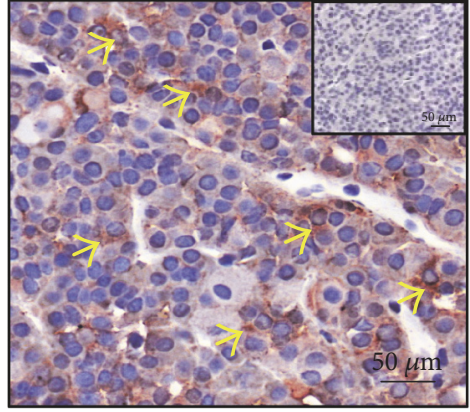

(a)

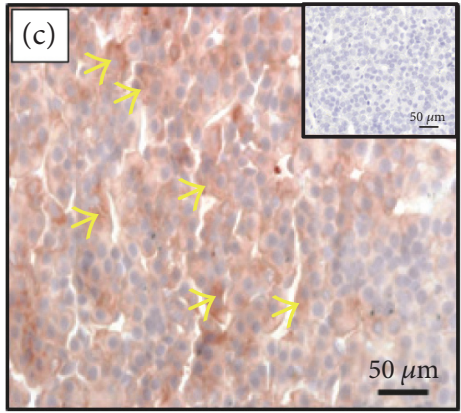

(c)

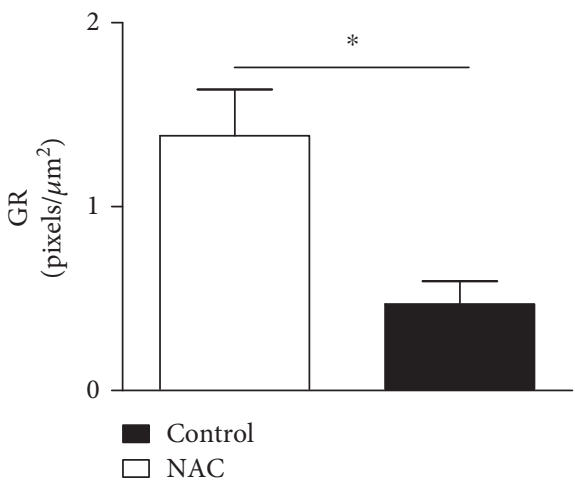

(e)

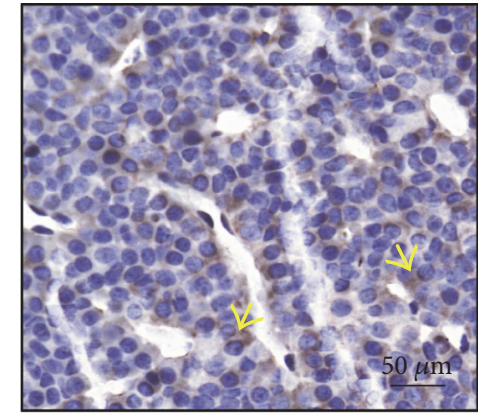

(b)

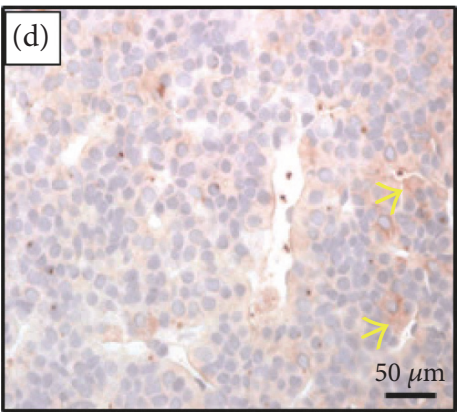

(d)

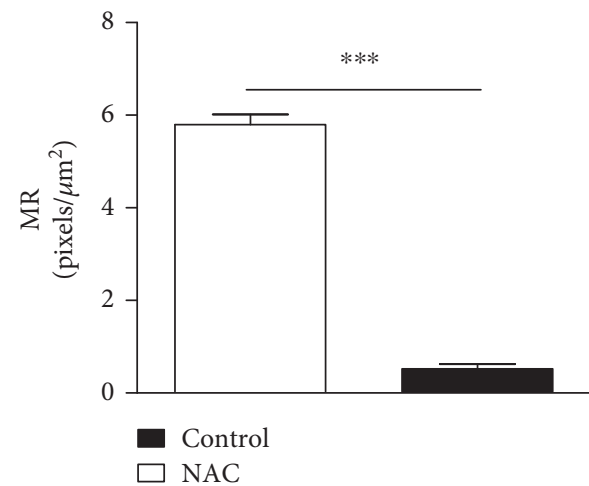

(f)

FIGURE 4: NAC decreases GR and MR expression in the anterior pituitary of Wistar rats. NAC (150 mg/kg, oral route) was given daily for 18 consecutive days and analysis was made by immunohistochemistry. The panels show representative photomicrographs of pituitary expression of GR in (a) control and (b) NAC-treated rats and MR in (c) control and (d) NAC-treated rats. The quantification of pixels associated with GR and MR expression is shown in (e) and (f), respectively. Inserts represent negative controls. Yellow arrows indicate immunolabelling of GR $(a, b)$ and MR $(c, d)$ in the anterior pituitary. Data are expressed as the mean \pm SEM of 6 animals. This result is a representative of two independent assays. ${ }^{*} p<0.05$ and ${ }^{* * *} p<0.001$.

is a precursor for reduced glutathione production, and scavenges oxidants directly, including hydroxyl radical, ${ }^{-} \mathrm{OH}$, and hypochlorous acid [8]; vitamin E is a peroxyl radical scavenger and due to its lipid solubility plays an important role in maintaining integrity of long-chain polyunsaturated fatty acids in the membranes of cells [6, 7]. The fact that prolonged treatment with two antioxidants with different mechanisms of action can increase circulating levels of corticosterone indicates that this is not an epiphenomenon, but suggests that inhibition of physiological levels of ROS in the HPA axis is responsible for its hyperactivity.

The HPA axis is the main neuroendocrine system that regulates responses to stress [8]. It is well known that the production of high levels of ROS into the glands that comprise the HPA axis is associated with the activation of a stressresponse system $[13,14]$. Therewith, antioxidant treatment reduces corticosterone levels in several models of diseases, including brain oxidative stress induced by lipopolysaccharide [26]. However, although accumulation of oxidative damage in cellular macromolecules is immensely toxic, ROS products by normal cell metabolism are vital to cell homeostasis maintenance $[15,16]$. Our hypothesis is that physiological levels of ROS have a fundamental role in maintaining the homeostasis of the HPA axis. In fact, treatment with NAC in normal rats effectively reduced ROS levels in chondrocytes and $\mathrm{Lin}^{-} \mathrm{CD} 45^{+} \mathrm{AnV} \mathrm{V}^{-}$marrow cells [27], suggesting that in our model, the antioxidant therapy probably reduces intracellular ROS content in the adrenal and pituitary glands. 


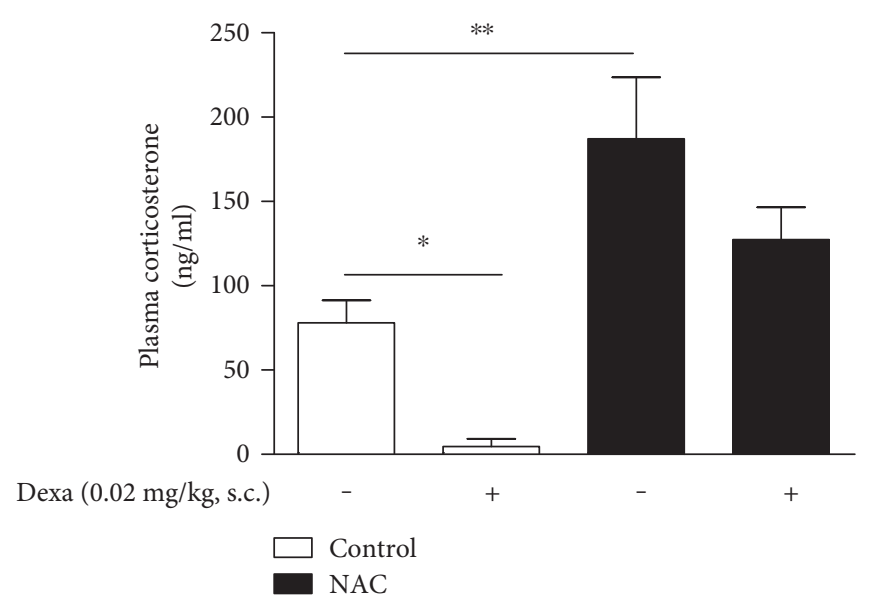

FIGURE 5: NAC impairs dexamethasone-induced negative feedback of the HPA axis in Wistar rats. NAC (150 mg/kg, oral route) was given daily for 18 consecutive days. Some groups of animals were injected with dexamethasone $(0.02 \mathrm{mg} / \mathrm{kg}$, s.c.) starting 13 days after the beginning of NAC treatment, daily during five consecutive days. Data are expressed as the mean \pm SEM of 5 animals. This result is a representative of two independent assays. ${ }^{*} p<0.05$ and ${ }^{* *} p<0.01$.

Thereby, we strongly suggest that any imbalance in the redox system in glands which comprise the HPA axis culminates in its hyperactivation.

In an attempt to elucidate how antioxidants induce the production of glucocorticoids by the HPA axis, we analyzed the expression of adrenal MC2R. Prolonged treatment with NAC increases adrenal MC2R expression. This higher adrenal MC2R expression after treatment can explain, at least partially, the capacity of NAC to increase circulating glucocorticoid levels. Increased expression of MC2R may lead to high activation of this receptor by ACTH and induction of the transcription of several key genes of enzymes involved in steroidogenesis, including StAR [28]. In fact, we showed that NAC induces an upregulation in the expression of StAR into the adrenal glands. StAR rapidly transports cholesterol to the inner mitochondrial membrane, where the conversion of this steroid precursor into pregnenolone, a precursor of steroid hormones, occurs [29]. This metabolic step is crucial to rapid glucocorticoid production into the adrenals in a stress stimulus, once steroidogenic cells store very little amount of glucocorticoids [10].

Although the increased expression of MC2R and StAR alone may explain the increase in glucocorticoids levels, other molecular alterations can also participate in the HPA axis hyperactivity noted after antioxidant treatment. Once the HPA axis is finely regulated by a negative feedback response on the hypothalamus and/or pituitary that normalizes circulating corticosterone levels, we hypothesized that antioxidants could induce a defect in the negative feedback regulation in the HPA axis. We observed that treatment with NAC downregulated the expression of Nrf2 in the anterior pituitary gland of rats. Nrf2 is a transcription factor that regulates expression of several antioxidant enzymes, including superoxide dismutase, catalase, glutathione peroxidase, and HO-1 [30]. Although NAC can induce upregulation of Nrf2 expression in phosgene-induced acute lung injury [31], our data is in accordance with others which described that
NAC inhibited Nrf2 expression in lymphoid malignant cell lines stimulated with honokiol [32], suggesting that the effect of antioxidant NAC on Nrf2 expression depends on the cell type and condition of the study. Furthermore, we noted that NAC also decreased the expression of HO-1 in the anterior pituitary of rats. The drop in HO-1 levels after treatment with NAC indicates that the low content of Nrf2 is associated with a reduced ability of this transcription factor to induce production of antioxidant enzymes by pituitary cells. We suppose that the downregulation of Nrf2 expression is a strategy of the organism to maintain homeostasis in rats treated for several days with NAC. In fact, it has been shown that exogenous antioxidants can reduce the expression and/or activity of endogenous antioxidant enzymes [33,34]. These data indicate that the pituitary as well as adrenals is also a direct target of antioxidant drug effects.

Our next approach was to investigate the sensitivity of the HPA axis to negative feedback induced by synthetic glucocorticoid in NAC-treated rats. Dexamethasone decreased plasma corticosterone levels in control rats; however, it did not alter circulating glucocorticoid amount in rats treated with NAC, showing that antioxidants abolish the ability of glucocorticoids to perform negative feedback of the HPA axis. NAC treatment also decreased expression of both GR and MR in the pituitary of rats, indicating that a reduction in glucocorticoid receptor expression in the pituitary of rats can explain the inability of dexamethasone to induce negative feedback of the HPA axis in NAC-treated animals. Our data confirmed the capacity of NAC in inhibiting GR expression, once NAC decreases GR protein levels in the hypothalamus of mice fed with a high-cholesterol diet [35].

Currently, many people consume dietary supplementation with antioxidants to combat diseases associated with aging [36]; however, several clinical trials testing benefits and harms of antioxidant supplements found that antioxidants have been unable to demonstrate beneficial effects and pointed that they seemed to cause an increase in all- 
cause mortality [37-40]. Once antioxidants induce a HPA axis dysfunction with concomitant increased levels of circulating glucocorticoids, this food supplement is shown as a risk to human health. This occurs because the hyperactivation of the HPA axis, and consequently the glucocorticoid signaling system, may alter the epigenetic landscape and influence genomic regulation and function conducting to the development of aging-related diseases [41]. Some harmful effects of hypercorticoidism, which can culminate with aging-related diseases, are deleterious effects on the central nervous system (CNS), including neuroinflammatory environment, loss of neuronal function, and apoptosis of neuronal cells, causing a decrease in hippocampal neurogenesis and an increase in neuroinflammation and neurodegeneration [42-44]. These deleterious effects of hypercorticoidism on the CNS can lead to the development of a variety of progressive neurodegenerative and psychiatric diseases, including schizophrenia, dementia, depression, Huntington's disease, and Alzheimer's disease [45-49]. Although we showed that antioxidants can induce high production of glucocorticoids, it is well known that chronic stress promotes redox imbalance throughout the body [50], as in blood of humans [51-53], such as in several structures of the CNS of rats including the frontal cortex, hypothalamus, and hippocampus [54]. These data suggest that chronic stress accelerates cellular aging through inducing increased levels of ROS [50]. These observations are conflicting with ours, once we show that antioxidants decreased the expression of Nrf2 and HO-1. However, the induction of ROS production is not the only mechanism related to stress-induced cellular aging. Chronic stress reduces brainderived neurotrophic factor (BDNF) in the hippocampus and prefrontal cortex and increases neuroinflammation, an alteration noted in the formation of depression [44]. Furthermore, chronic stress induces prolonged periods of glutamate release in the hippocampus and decreases the ability to clear extracellular glutamate. These alterations in the glutamate transmission may be related to the impairments in the spatial and contextual memory performance and stress-associated psychiatric disorders, including mood and anxiety [55].

In addition, hypercorticoidism can also increase susceptibility to cancer [56], one of the most important aging-related diseases. Although ROS can cause oncogenic mutations and activate oncogenic pathways [57], dietary supplementation with antioxidants promotes increased incidence and death from lung and prostate cancer [58]. Furthermore, antioxidants induce melanoma progression by promoting metastasis [59]. One possibility is that the high incidence of cancer in people which use dietary supplementation with antioxidants can be related to hyperactivation of the HPA axis.

In summary, our results indicate that antioxidant therapy can induce an activation of the HPA axis, with an increase in the levels of systemic glucocorticoids by upregulating ACTH receptor in the adrenal and downregulating glucocorticoid receptors in the pituitary. Thereby, indiscriminate use of antioxidant supplements can be a risk to develop several morbidities related to persistent hypercorticoidism, as observed in Cushing's disease.

\section{Conflicts of Interest}

The authors declare that they have no conflict of interest. Furthermore, the mentioned received funding in the "Acknowledgments" section did not lead to any conflict of interests regarding the publication of this manuscript.

\section{Acknowledgments}

The authors thank Mr. Andrey Junior Moreira Fernandes from the FIOCRUZ for his technical support. This work was supported by grants from the Conselho Nacional de Desenvolvimento Científico and Tecnológico (CNPq) MCT/CNPq/CT-SAÚDE/MS/SCTIE/DECIT no. 42/2010, Fundação Carlos Chagas de Amparo à Pesquisa do Estado do Rio de Janeiro (FAPERJ), Programa de Auxílio à Pesquisa (PAPESVI/FIOCRUZ), and Ministério da Saúde, Brazil.

\section{References}

[1] B. Kalyanaraman, "Teaching the basics of redox biology to medical and graduate students: oxidants, antioxidants and disease mechanisms," Redox Biology, vol. 1, no. 1, pp. 244257, 2013.

[2] Q. Ma, "Role of nrf2 in oxidative stress and toxicity," Annual Review of Pharmacology and Toxicology, vol. 53, no. 1, pp. 401-426, 2013.

[3] S. Kardeh, S. Ashkani-Esfahani, and A. M. Alizadeh, "Paradoxical action of reactive oxygen species in creation and therapy of cancer," European Journal of Pharmacology, vol. 735, no. 1, pp. 150-168, 2014.

[4] M. Nordgren and M. Fransen, "Peroxisomal metabolism and oxidative stress," Biochimie, vol. 98, pp. 56-62, 2014.

[5] K. B. Narayanan and H. H. Park, "Pleiotropic functions of antioxidant nanoparticles for longevity and medicine," Advances in Colloid and Interface Science, vol. 201-202, pp. 30-42, 2013.

[6] H. J. Forman, O. Augusto, R. Brigelius-Flohe et al., "Even free radicals should follow some rules: a guide to free radical research terminology and methodology," Free Radical Biology \& Medicine, vol. 78, pp. 233-235, 2015.

[7] M. G. Traber and J. Atkinson, "Vitamin E, antioxidant and nothing more," Free Radical Biology \& Medicine, vol. 43, no. 1, pp. 4-15, 2007.

[8] O. Dean, F. Giorlando, and M. Berk, "N-acetylcysteine in psychiatry: current therapeutic evidence and potential mechanisms of action," Journal of Psychiatry \& Neuroscience, vol. 36, no. 2, pp. 78-86, 2011.

[9] C. A. Van Hulle, E. A. Shirtcliff, K. Lemery-Chalfant, and H. H. Goldsmith, "Genetic and environmental influences on individual differences in cortisol level and circadian rhythm in middle childhood," Hormones and Behavior, vol. 62, no. 1, pp. 36-42, 2012.

[10] T. M. Buckley and A. F. Schatzberg, "On the interactions of the hypothalamic-pituitary-adrenal (HPA) axis and sleep: normal HPA axis activity and circadian rhythm, exemplary sleep disorders," The Journal of Clinical Endocrinology and Metabolism, vol. 90, no. 5, pp. 3106-3114, 2005.

[11] Y. M. Ulrich-Lai and J. P. Herman, "Neural regulation of endocrine and autonomic stress responses," Nature Reviews Neuroscience, vol. 10, no. 6, pp. 397-409, 2009. 
[12] T. Frodl and V. O'Keane, "How does the brain deal with cumulative stress? A review with focus on developmental stress, HPA axis function and hippocampal structure in humans," Neurobiology of Disease, vol. 52, pp. 24-37, 2013.

[13] A. Papadimitriou and K. N. Priftis, "Regulation of the hypothalamic-pituitary-adrenal axis," Neuroimmunomodulation, vol. 16, no. 5, pp. 265-271, 2009.

[14] T. Kino, "Stress, glucocorticoid hormones, and hippocampal neural progenitor cells: implications to mood disorders," Frontiers in Physiology, vol. 6, p. 230, 2015.

[15] M. E. Keller-Wood and M. F. Dallman, "Corticosteroid inhibition of ACTH secretion," Endocrine Reviews, vol. 5, no. 1, pp. 1-24, 1984.

[16] J. S. Seo, J. Y. Park, J. Choi et al., "NADPH oxidase mediates depressive behavior induced by chronic stress in mice," The Journal of Neuroscience, vol. 32, no. 28, pp. 9690-9699, 2012.

[17] K. Asaba, Y. Iwasaki, M. Yoshida et al., "Attenuation by reactive oxygen species of glucocorticoid suppression on proopiomelanocortin gene expression in pituitary corticotroph cells," Endocrinology, vol. 145, no. 1, pp. 39-42, 2004.

[18] J. Kanta, "The role of hydrogen peroxide and other reactive oxygen species in wound healing," Acta Medica, vol. 54, no. 3, pp. 97-101, 2011.

[19] W. Bild, A. Ciobica, M. Padurariu, and V. Bild, "The interdependence of the reactive species of oxygen, nitrogen, and carbon," Journal of Physiology and Biochemistry, vol. 69, no. 1, pp. 147-154, 2013.

[20] F. S. Rodrigues, M. A. Souza, D. V. Magni et al., "N-acetylcysteine prevents spatial memory impairment induced by chronic early postnatal glutaric acid and lipopolysaccharide in rat pups," PloS One, vol. 8, no. 10, Article ID e78332, 2013.

[21] Z. J. Zhang, Y. H. Qian, H. T. Hu, J. Yang, and G. D. Yang, "The herbal medicine Dipsacus asper wall extract reduces the cognitive deficits and overexpression of beta-amyloid protein induced by aluminum exposure," Life Sciences, vol. 73, no. 19, pp. 2443-2454, 2003.

[22] J. B. da Silva, S. Temponi Vdos, C. M. Gasparetto et al., "Vernonia condensata Baker (Asteraceae): a promising source of antioxidants," Oxidative Medicine and Cellular Longevity, vol. 2013, Article ID 698018, 9 pages, 2013.

[23] O. Chan, K. Inouye, M. Vranic, and S. G. Matthews, "Hyperactivation of the hypothalamo-pituitary-adrenocortical axis in streptozotocin-diabetes is associated with reduced stress responsiveness and decreased pituitary and adrenal sensitivity," Endocrinology, vol. 143, no. 5, pp. 1761-1768, 2002.

[24] C. Wotus, B. K. Levay-Young, L. M. Rogers, C. E. GomezSanchez, and W. C. Engeland, "Development of adrenal zonation in fetal rats defined by expression of aldosterone synthase and 11beta-hydroxylase," Endocrinology, vol. 139, no. 10, pp. 4397-4403, 1998.

[25] I. C. Alexandreanu and D. M. Lawson, "Heme oxygenase in the rat anterior pituitary: immunohistochemical localization and possible role in gonadotropin and prolactin secretion," Experimental Biology and Medicine, vol. 228, no. 1, pp. 6469, 2003

[26] A. A. Kheir-Eldin, T. K. Motawi, M. Z. Gad, and H. M. Abd-ElGawad, "Protective effect of vitamin E, betacarotene and $\mathrm{N}$-acetylcysteine from the brain oxidative stress induced in rats by lipopolysaccharide," The International Journal of Biochemistry \& Cell Biology, vol. 33, no. 5, pp. 475-482, 2001.
[27] Y. Y. Jang and S. J. Sharkis, "A low level of reactive oxygen species selects for primitive hematopoietic stem cells that may reside in the low-oxygenic niche," Blood, vol. 110, no. 8, pp. 3056-3063, 2007.

[28] J. N. Winnay and G. D. Hammer, "Adrenocorticotropic hormone-mediated signaling cascades coordinate a cyclic pattern of steroidogenic factor 1-dependent transcriptional activation," Molecular Endocrinology, vol. 20, no. 1, pp. 147$166,2006$.

[29] W. L. Miller, "Steroidogenic acute regulatory protein (StAR), a novel mitochondrial cholesterol transporter," Biochimica et Biophysica Acta, vol. 1771, no. 6, pp. 663-676, 2007.

[30] B. Li, S. Liu, L. Miao, and L. Cai, "Prevention of diabetic complications by activation of Nrf2: diabetic cardiomyopathy and nephropathy," Experimental Diabetes Research, vol. 2012, p. 216512, 2012.

[31] S. Ling, J. Duan, R. Ni, and J. W. Xu, “2,3,5,4'-Tetrahydroxystilbene-2-O-beta-D-glucoside promotes expression of the longevity gene klotho," Oxidative Medicine and Cellular Longevity, vol. 2016, Article ID 3128235, 11 pages, 2016.

[32] D. Q. Gao, S. Qian, and T. Ju, “Anticancer activity of honokiol against lymphoid malignant cells via activation of ROS-JNK and attenuation of Nrf2 and NF-kappaB," Journal of BUON, vol. 21, no. 3, pp. 673-679, 2016.

[33] K. M. Pires, M. Lanzetti, C. R. Rueff-Barroso et al., "Oxidative damage in alveolar macrophages exposed to cigarette smoke extract and participation of nitric oxide in redox balance," Toxicology in Vitro, vol. 26, no. 6, pp. 791-798, 2012.

[34] J. K. Kim, J. Park, T. H. Ryu, and M. Nili, "Effect of N-acetyl-1cysteine on Saccharomyces cerevisiae irradiated with gammarays," Chemosphere, vol. 92, no. 5, pp. 512-516, 2013.

[35] L. M. Korou, G. Agrogiannis, C. Koros et al., "Impact of Nacetylcysteine and sesame oil on lipid metabolism and hypothalamic-pituitary-adrenal axis homeostasis in middleaged hypercholesterolemic mice," Scientific Reports, vol. 4, p. $6806,2014$.

[36] G. C. Domenico Fusco, M. R. L. Monaco, and M. Cesari, "Effects of antioxidant supplementation on the aging process," Clinical Interventions in Aging, vol. 2, no. 3, pp. 377-387, 2007.

[37] G. Bjelakovic, D. Nikolova, R. G. Simonetti, and C. Gluud, "Antioxidant supplements for prevention of gastrointestinal cancers: a systematic review and meta-analysis," Lancet, vol. 364, no. 9441, pp. 1219-1228, 2004.

[38] G. Bjelakovic, D. Nikolova, L. L. Gluud, R. G. Simonetti, and C. Gluud, "Antioxidant supplements for prevention of mortality in healthy participants and patients with various diseases," The Cochrane Database of Systematic Reviews, vol. 3, no. 3, p. CD007176, 2012.

[39] E. R. Miller 3rd, R. Pastor-Barriuso, D. Dalal, R. A. Riemersma, L. J. Appel, and E. Guallar, "Meta-analysis: high-dosage vitamin E supplementation may increase all-cause mortality," Annals of Internal Medicine, vol. 142, no. 1, pp. 37-46, 2005.

[40] H. Macpherson, A. Pipingas, and M. P. Pase, "Multivitaminmultimineral supplementation and mortality: a meta-analysis of randomized controlled trials," The American Journal of Clinical Nutrition, vol. 97, no. 2, pp. 437-444, 2013.

[41] N. C. Gassen, G. P. Chrousos, E. B. Binder, and A. S. Zannas, "Life stress, glucocorticoid signaling, and the aging epigenome: implications for aging-related diseases," Neuroscience and Biobehavioral Reviews, vol. 74, no. Part B, pp. 356-365, 2017. 
[42] F. Jeanneteau, M. J. Garabedian, and M. V. Chao, "Activation of Trk neurotrophin receptors by glucocorticoids provides a neuroprotective effect," Proceedings of the National Academy of Sciences of the United States of America, vol. 105, no. 12, pp. 4862-4867, 2008.

[43] P. J. Lucassen, J. Pruessner, N. Sousa et al., "Neuropathology of stress," Acta Neuropathologica, vol. 127, no. 1, pp. 109-135, 2014.

[44] A. M. Hemmerle, J. P. Herman, and K. B. Seroogy, "Stress, depression and Parkinson's disease," Experimental Neurology, vol. 233, no. 1, pp. 79-86, 2012.

[45] A. Machado, A. J. Herrera, R. M. de Pablos et al., "Chronic stress as a risk factor for Alzheimer's disease," Reviews in the Neurosciences, vol. 25, no. 6, pp. 785-804, 2014.

[46] C. D. Pandya, A. Crider, and A. Pillai, "Glucocorticoid regulates parkin expression in mouse frontal cortex: implications in schizophrenia," Current Neuropharmacology, vol. 12, no. 2, pp. 100-107, 2014.

[47] A. A. Hubers, R. C. van der Mast, A. M. Pereira et al., "Hypothalamic-pituitary-adrenal axis functioning in Huntington's disease and its association with depressive symptoms and suicidality," Journal of Neuroendocrinology, vol. 27, no. 3, pp. 234-244, 2015.

[48] Z. Bhagwagar, S. Hafizi, and P. J. Cowen, "Increased salivary cortisol after waking in depression," Psychopharmacology, vol. 182, no. 1, pp. 54-57, 2005.

[49] E. K. Adam, L. D. Doane, R. E. Zinbarg, S. Mineka, M. G. Craske, and J. W. Griffith, "Prospective prediction of major depressive disorder from cortisol awakening responses in adolescence," Psychoneuroendocrinology, vol. 35, no. 6, pp. 921-931, 2010.

[50] E. Miller, A. Morel, L. Saso, and J. Saluk, "Isoprostanes and neuroprostanes as biomarkers of oxidative stress in neurodegenerative diseases," Oxidative Medicine and Cellular Longevity, vol. 2014, Article ID 572491, 10 pages, 2014.

[51] L. Cohen, G. D. Marshall Jr., L. Cheng, S. K. Agarwal, and Q. Wei, "DNA repair capacity in healthy medical students during and after exam stress," Journal of Behavioral Medicine, vol. 23, no. 6, pp. 531-544, 2000.

[52] A. Nakhaee, F. Shahabizadeh, and M. Erfani, "Protein and lipid oxidative damage in healthy students during and after exam stress," Physiology \& Behavior, vol. 118, pp. 118-121, 2013.

[53] M. Sivonova, I. Zitnanova, L. Hlincikova, I. Skodacek, J. Trebaticka, and Z. Durackova, "Oxidative stress in university students during examinations," Stress, vol. 7, no. 3, pp. 183-188, 2004.

[54] G. Lucca, C. M. Comim, S. S. Valvassori et al., "Increased oxidative stress in submitochondrial particles into the brain of rats submitted to the chronic mild stress paradigm," Journal of Psychiatric Research, vol. 43, no. 9, pp. 864-9, 2009.

[55] M. Popoli, Z. Yan, B. S. McEwen, and G. Sanacora, "The stressed synapse: the impact of stress and glucocorticoids on glutamate transmission," Nature Reviews Neuroscience, vol. 13, no. 1, pp. 22-37, 2011.

[56] S. Azher, O. Azami, C. Amato, M. McCullough, A. Celentano, and N. Cirillo, "The non-conventional effects of glucocorticoids in cancer," Journal of Cellular Physiology, vol. 231, no. 11, pp. 2368-2373, 2016.
[57] C. Gorrini, I. S. Harris, and T. W. Mak, "Modulation of oxidative stress as an anticancer strategy," Nature Reviews Drug Discovery, vol. 12, no. 12, pp. 931-947, 2013.

[58] E. A. Klein, I. M. Thompson Jr., C. M. Tangen et al., "Vitamin $\mathrm{E}$ and the risk of prostate cancer: the Selenium and Vitamin $\mathrm{E}$ Cancer Prevention Trial (SELECT)," JAMA, vol. 306, no. 14, pp. 1549-1556, 2011.

[59] E. Piskounova, M. Agathocleous, M. M. Murphy et al., "Oxidative stress inhibits distant metastasis by human melanoma cells," Nature, vol. 527, no. 7577, pp. 186-191, 2015. 


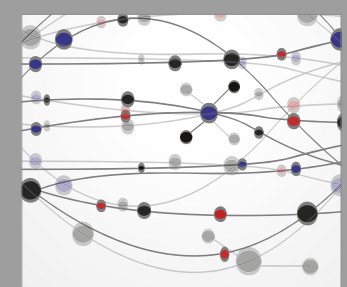

The Scientific World Journal
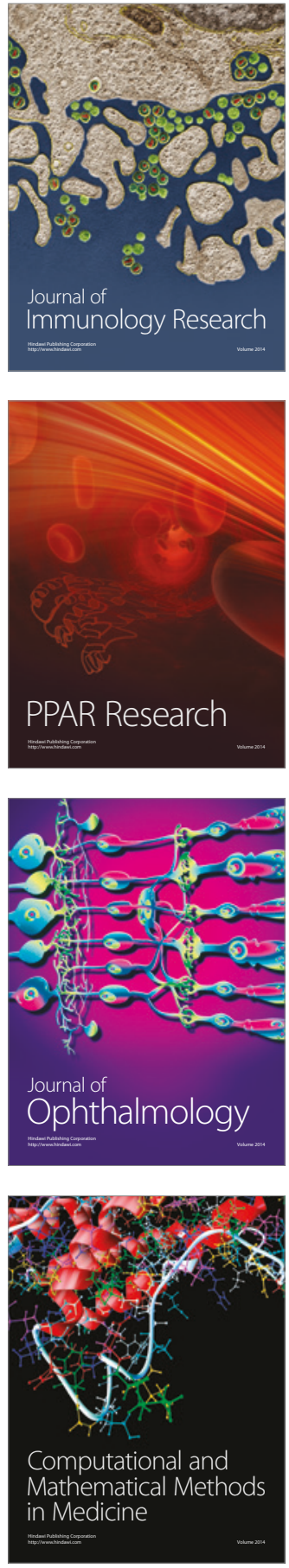

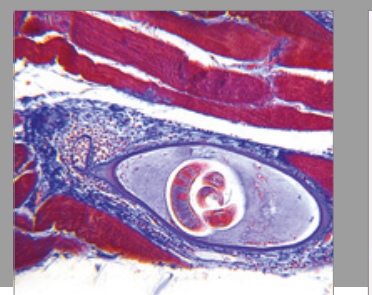

Gastroenterology Research and Practice
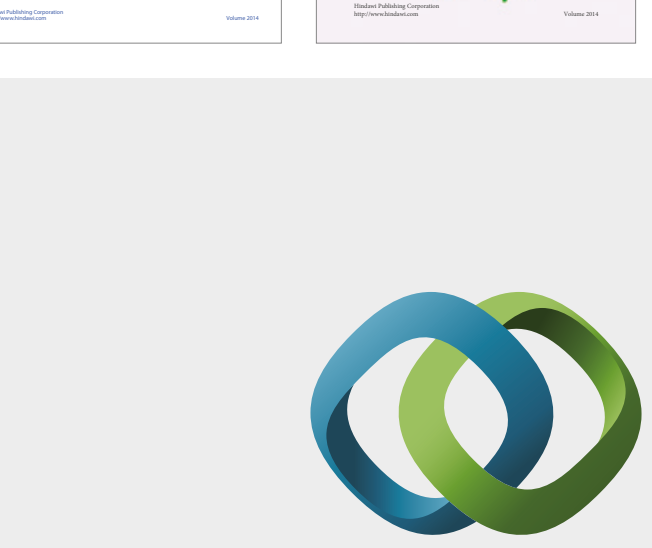

\section{Hindawi}

Submit your manuscripts at

https://www.hindawi.com
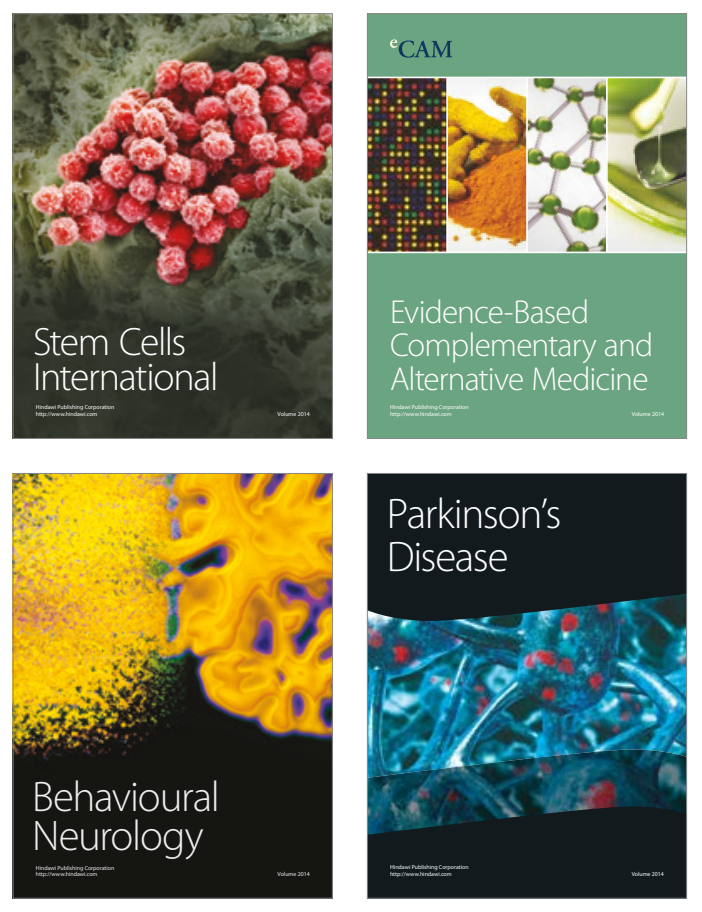
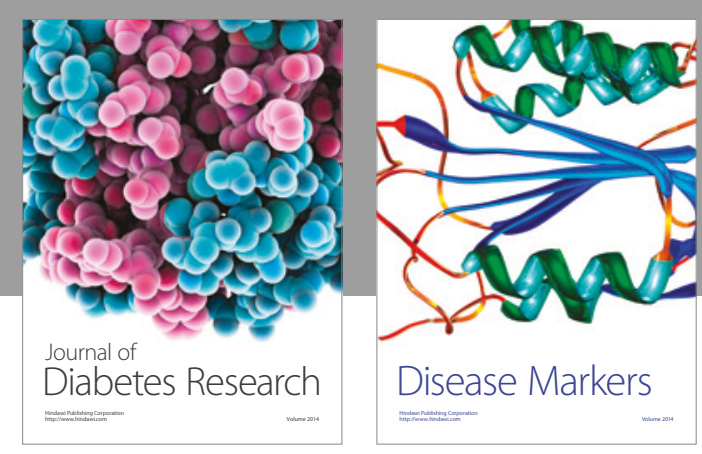

Disease Markers
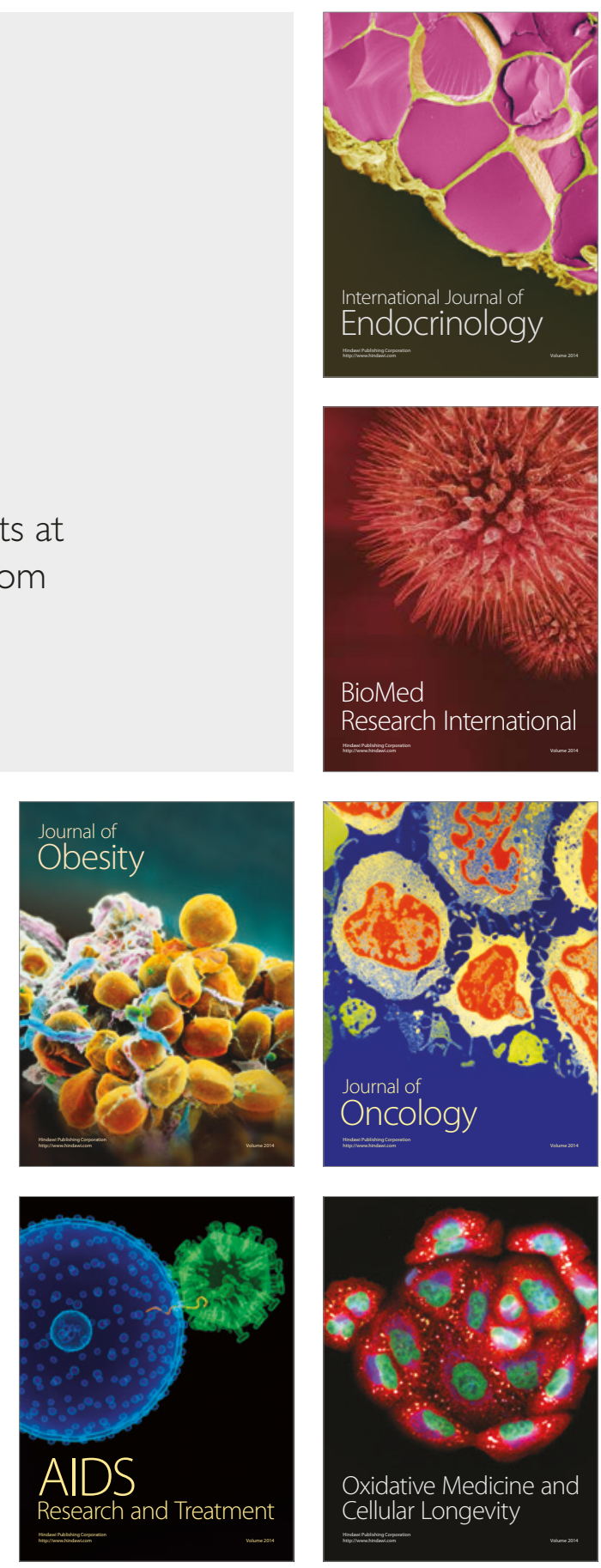